\title{
REVIEW
}

\section{Neurostimulation treatment for angina pectoris}

\author{
S Murray, P D Collins, M A James
}

Despite the wealth of treatments available for the management of angina pectoris, a significant proportion of patients remain refractory. These patients seem to be survivors, so that an individual with refractory angina may suffer with it for many years; consequently patients with refractory angina pectoris usually require multiple admissions, often to expensive cardiac units. ${ }^{1}$ Recently transmyocardial laser revascularisation (TMLR) has enjoyed considerable world wide popularity as a potential treatment strategy. However, the recently published TMLR trial from Papworth Hospital led the investigators to conclude that they cannot advocate the adoption of TMLR for the management of refractory angina. ${ }^{2}$ Neurostimulation presents an alternative treatment strategy for which efficacy data continues to grow, although there has not yet been a large randomised controlled trial into its use in the treatment of refractory angina.

This article summarises neurostimulation research to date and reviews the current theories on the mechanisms of action.

\section{Development of neurostimulation for refractory angina}

Neurostimulation was developed in response to the gate theory of pain transmission to provide a non-pharmacological method of providing pain relief. ${ }^{3}$ Despite initial excitement, the effects were found to be variable. In the case of peripheral vascular disease, neurostimulation in the form of transcutaneous electrical nerve stimulation (TENS) and spinal cord stimulation (SCS) was found to be particularly useful. ${ }^{4}$ As well as providing pain relief, neurostimulation also improved microcirculatory blood flow, and led to ischaemic ulcer healing.

In response to the work in peripheral vascular disease, other workers began to look for a similar effect in different ischaemic conditions, with Mannheimer and colleagues first reporting the success of TENS in patients with chronic intractable angina pectoris. ${ }^{5}$ They showed that TENS not only reduced patients' symptoms, but also increased the myocardium's threshold for ischaemia. ${ }^{6}$ The technique was applied clinically only in patients where medical and surgical treatment was failing, as there was concern that "masking" ischaemic symptoms was potentially dangerous - that is, that TENS was removing an important "warning mechanism".

SCS was first used by Murphy and Giles to treat angina in 1987, ${ }^{7}$ after Mannheimer's work with TENS. ${ }^{56}$ Since then both modalities have been researched in a clinical setting with patients suffering from refractory angina pectoris; this work is reviewed below.

\section{Evidence of efficacy}

Following Mannheimer's initial work, there have been many studies investigating the effects of neurostimulation using both TENS and SCS. All studies have suggested an antiischaemic effect as assessed on exercise testing, ${ }^{8-11}$ ambulatory ECG ${ }^{11}{ }^{12}$ stress echocardiography, ${ }^{13}$ and invasive measurement of lactate from coronary sinus blood samples. ${ }^{6} 914$ Patients have also reported significantly fewer angina attacks, with decreased consumption of glyceryl trinitrate when using SCS or TENS (although these cannot be regarded as objective measures of ischaemia, rather a reflection of symptoms). As well as this anti-ischaemic effect, neurostimulation has an apparent benefit even after the period of stimulation. This so called "carry over" effect was first demonstrated in the context of refractory angina by Sanderson and colleagues. ${ }^{9}$ They studied SCS patients using an experimental design which included a control phase, followed by the treatment phase before a second control phase. This second control phase showed a significant improvement over the first, although the greatest effect was still observed during the treatment phase. Whether this effect is real or the result of a confounding factor, such as ischaemic preconditioning is unknown. As yet, there are no trials repeating these protocols in a randomised order. There is additional evidence that neurostimulation might have benefits beyond the period of stimulation from ambulatory ECG data. Sanderson and colleagues ${ }^{11}$ and DeJongste and colleagues ${ }^{12}$ have shown a decrease in total ischaemic burden with SCS over a 48 hour control period; this occurs with stimulation for one hour three times per day. In the interim, ischaemia appears to be prevented. In clinical practice, there is anecdotal evidence to provide support that routine use of the device leads to a decrease in angina attacks throughout the day. This therapeutic efficacy is reflected in a reduction in acute admissions, which has been observed over a period of years. ${ }^{1}$ However, the concern that neurostimulation may be dangerous is not borne out; it does not conceal myocardial infarction or unstable angina, ${ }^{11} 15$ and there is no evidence of proarrhythmia during ambulatory ECG. ${ }^{12}$ 


\section{Mechanisms of action}

At present the exact mechanism of action of neurostimulation is not known. All of the following mechanisms have been invoked, and it is possible that more than one of them is responsible for the results of neurostimulation.

PLACEBO EFFECT

The placebo response is the psychological effect any form of intervention has upon a patient which is unrelated to its physiological action. A placebo treatment is primarily physiologically inert. This effect is estimated to contribute up to $30-40 \%$ of the initial benefit of any medical treatment, ${ }^{16}$ but these effects diminish with time, so that any placebo effect is negligible after 2-3 months. ${ }^{17}$ There are particular problems in clinical trials with neurostimulation with regard to controlling for placebo effects; as yet there is no satisfactory method for using a placebo control. Both TENS and SCS produce a characteristic paraesthesia in the area of stimulation, which the patient is plainly aware of. The most acceptable placebo control method to date is the use of "sham TENS"; this involves the use of a TENS unit attached to the patient via an oscilloscope, which is in clear view of the patient. The device also has a light that signifies it is active. When the unit is switched on, the light comes on and a wave form appears upon the oscilloscope which can be clearly seen by the patient. However, no current is ever delivered to the patient. This method controls for the effect of using the device as treatment, but cannot allow for the effect of feeling stimulation. The use of sham TENS is therefore only of value in single sitting experiments using volunteers naive to neurostimulation. However, in clinical trials in which a crossover protocol is most often used, owing to the relatively small number of study patients available, it seems likely that the patient will quickly differentiate between placebo and treatment.

Another method of controlling for placebo effect with SCS was employed by Hautvast and colleagues. ${ }^{18}$ They used a prospective, randomised trial in which subjects were randomised to treatment or control groups. Both the treatment and control groups had SCS units implanted at the start of the study, but the controls did not have the units activated until after six weeks. In other words, one group was implanted with "sham SCS" for six weeks. Thus these researchers evaluated the placebo response of the implantation procedure itself. Their results showed a significant response in the active SCS group as compared to the sham SCS group, with improvements in ischaemic parameters on treadmill testing and ambulatory ECG, decreased angina attacks, glyceryl trinitrate consumption, and an improvement in quality of life.

While the placebo response can have profound effects on a patient's wellbeing, it is thought that the effect cannot influence ischaemia upon objective assessment. ${ }^{19}$ Measures such as glyceryl trinitrate consumption, number of angina attacks, and maximal exercise times on exercise testing are not wholly objective. However, objective assessments have been made using ST segment analysis during exercise, stress echocardiography, and invasive measurements of coronary sinus lactate concentrations. It is extremely unlikely that the placebo effect could account for these results. Khurmi and colleagues reported that placebos do not influence objective measures of ischaemia, such as ST segment changes. ${ }^{19}$ Furthermore, in our clinical experience in this centre patients implanted up to 10 years previously still derive benefit from their neurostimulators, ${ }^{1}$ which is notably longer than the anticipated duration of any placebo effect.

\section{PRIMARY ANTINOCICEPTIVE EFFECT}

TENS and SCS were created in response to the gate theory of pain. Their primary action was to mask pain by producing a counterstimulation, and it is this notion which has led to resistance by many clinicians to use neurostimulation in angina pectoris. However, the historical development of their use in angina arose from the observed anti-ischaemic effects in peripheral vascular disease. Despite this, further scepticism grew from the work by Chandler and colleagues in $1993 .{ }^{20}$ This group looked at the effects of neurostimulation on the spinal pathways and central nervous systems of monkeys. Specifically they measured extracellular potentials in spinothalamic tract neurones in response to experimentally produced painful stimuli (intracardiac injections of bradykinin) with and without concurrent SCS. The SCS parameters were similar to those used by Mannheimer and colleagues. ${ }^{56}$ They found that SCS could indeed block transmission of painful stimuli, and concluded that SCS could deprive patients "of an important warning signal". However, the group makes no attempt to consider the evidence for an anti-ischaemic effect, despite citing the work in the introduction to this study. Indeed their report implicitly suggests that the mechanism they describe accounts for the whole effect of SCS as observed in clinical practice.

In considering the relation between pain and ischaemia in angina, it becomes clear that the two are closely associated. Myocardial ischaemia involves a "vicious circle" as pain and distress lead to both a general and segmental increase of sympathetic nervous activity, producing further myocardial oxygen demand. ${ }^{21}$ Indeed psychological distress in itself is known to be able to initiate an attack of angina pectoris. Thus, by simply alleviating pain could it be possible to break this circle and produce a reduction in ischaemia? The ambulatory ECG studies from Sanderson and colleagues ${ }^{11}$ and DeJongste and colleagues ${ }^{12}$ have confirmed that neurostimulation is not only associated with a decrease in angina attacks, but also a fall in total ischaemic burden. These studies have also shown a decrease in attacks in between periods of stimulation, suggesting that the effect persists longer than the period of active stimulation. Also, if we look again at Mannheimer's invasive work using coronary sinus sampling, we see not a masking of symptoms, but a shift in the ischaemic threshold, with symptoms appearing as before, but at a 
higher level of exertion and corresponding with lactate production. ${ }^{6}{ }^{14}$

While the relief of pain during an angina attack is likely to be important in resolving ongoing ischaemia, it is unlikely that this mechanism is the single mode of action of TENS and SCS.

ROLE OF ENDOGENOUS OPIATES

Experimental work from Mannheimer's team has suggested that the heart is capable of producing endogenous opioids, and there has been recent interest in $\beta$ endorphin, which may be released during SCS in angina. ${ }^{22}$ This study looked at concentrations of $\beta$ endorphin during control right atrial pacing, followed by right atrial pacing during SCS; individuals demonstrated varied results, although the group did show a mean increase in $\beta$ endorphin release during SCS. Once again, the study was non-randomised. The authors suggest that the opioids may act by antagonising sympathetic stimulation, so reducing contractility and hence oxygen consumption. However, these results must be interpreted cautiously. Firstly, the same group found that naloxone did not antagonise the beneficial effects of neurostimulation in a similar stress pacing protocol. ${ }^{23}$ Furthermore, $\beta$ endorphin concentrations have been linked to silent ischaemia, and not abolition of ischaemia. ${ }^{24}$ Certainly, opiates are widely used to treat acute ischaemic pain, and there is a reported case of using continuous intrathecal opiate to treat refractory angina. ${ }^{25}$ Whether pain relief is able to produce a decrease in myocardial oxygen consumption is not clear, although it is feasible.

INCREASE IN CORONARY FLOW

Work in peripheral vascular disease has shown that neurostimulation leads to an increase in oxygen delivery at the microcirculatory level. ${ }^{4}$ Could a similar mechanism be present in the heart? In 1994 Chauhan and colleagues examined the effects of TENS upon coronary artery flow within three groups of patients: chronic angina sufferers with a single diseased vessel; syndrome X patients; and cardiac transplant patients. ${ }^{26}$ They examined coronary flow using an intravascular Doppler catheter sited in a normal coronary artery. Their results showed that TENS produced an increase in flow at rest in both the angina and syndrome $\mathrm{X}$ patients, but not in the transplant group, which suggested a neurally mediated action of TENS. However, this study examined the effects of TENS at rest in normal coronary arteries. Repeat studies on syndrome $\mathrm{X}$ patients by Sanderson and colleagues $^{27}$ and angina sufferers by DeJongste and colleagues ${ }^{28}$ have failed to show an increase in flow; indeed, in diseased vessels flow rates remain the same or actually fall with TENS.

We must also consider the effect of an increase in flow at rest; coronary flow is proportionally related to oxygen consumption, ${ }^{29}$ and we could therefore interpret any flow increase at rest to be caused by increased oxygen demand. Conversely, the fall in coronary flow observed could represent a reduction in oxygen demand, although this effect did not achieve significance. Finally, positron emission tomography (PET) studies by Hautvaust and colleagues $^{30}$ and DeLandsheere and colleagues ${ }^{31}$ did not show an increase in myocardial flow with SCS. It is therefore unlikely that neurostimulation works through this mechanism.

ANTISYMPATHETIC NERVOUS SYSTEM EFFECT Mannheimer's group have long held the view that neurostimulation acts similarly to $\beta$ adrenoreceptor blocking drugs, leading to decreased oxygen consumption, and reflected by a fall in rate-pressure product. Several investigators have shown a modest fall in systemic systolic pressures during TENS and SCS. ${ }^{32} 33$ There is also a correlation with the surgical option of cervical sympathectomy in intractable angina; this too has been shown to increase ischaemic threshold, and not simply mask pain. ${ }^{34}$ Finally, SCS treatment in peripheral vascular disease has been shown to achieve its beneficial vasomotor effects via an antisympathetic mechanism. Thus the antisympathetic mechanism of action was an attractive theory with regard to angina treatment. Sanderson and colleagues used TENS in healthy volunteers and performed standard autonomic reflex tests in an attempt to establish a link between TENS and an antisympathetic mode of action. ${ }^{35}$ The results showed only a minor antisympathetic response, and the individual results of the volunteers were inconsistent (suggesting the possibility that some volunteers were "responders" and others "nonresponders" who actually increase sympathetic tone in response to TENS).

Mannheimer's group has recently published data using noradrenaline (norepinephrine) spillover techniques in order to evaluate both myocardial and overall sympathetic tone in angina patients with SCS implants. ${ }^{36}$ The results indicated that there was little effect from SCS in reducing cardiac sympathetic activity, although overall sympathetic activity was decreased. This could be secondary to a reduction in ischaemia, and hence angina, rather than a primary event - that is, it could reflect a break in the "vicious cycle" from obtaining analgesia. However, they could not provide evidence of a direct antisympathetic effect upon the myocardium, although it is possible that the technique used is not sensitive enough to detect subtle changes.

REDISTRIBUTION OF MYOCARDIAL BLOOD FLOW The results on angina in response to neurostimulation are similar to those for the effect of theophyllines in angina treatment; ischaemic threshold is increased without increasing coronary flow, or even decreasing it by reducing myocardial oxygen consumption. ${ }^{37}{ }^{38}$ Theophylline is thought to act by blocking adenosine mediated intramural steal and subendocardial steal phenomena. Thus, it has been suggested that blood is redistributed from non-ischaemic areas to ischaemic ones, leading to the term "Robin Hood effect". PET studies from Hautvaust and colleagues have suggested that SCS may lead to a "homogenisation" of flow throughout the myocardium without actually 
increasing net peak flows at exercise. ${ }^{30}$ Thus SCS may also be acting as an adenosine antagonist. Indeed, Hautvaust and colleagues used dipyridamole (which potentiates endogenous adenosine) in their PET protocol, and make the comment that SCS appears to attenuate its effect. ${ }^{30}$ Furthermore, a study of the effects of SCS on left ventricular function during stress echocardiography, which used adenosine as a pharmacological stressor, reported that SCS was associated with tolerance of higher adenosine concentrations infused. ${ }^{13}$ Thus neurostimulation could produce the "Robin Hood effect" to achieve its benefits by attenuating the effects of adenosine. This "redistribution mechanism" could result in a fundamental difference in response between sufferers of chronic stable angina and those with refractory angina, which predisposes them to a more beneficial response to SCS owing to the increased incidence of collateral coronary vessels. The incidence of collaterals in the coronary circulation is higher in those with more severe disease, and their development is not related to exercise or training $^{39}$; it is also known that collateral vessels are much more sensitive to adenosine mediated steal phenomena, and so in patients with refractory angina any adenosine blocking effect of SCS might be particularly important. ${ }^{40}$ By the same virtue, one might not expect to find a benefit with neurostimulation in patients without collaterals.

\section{Summary}

Neurostimulation is a safe and effective treatment for refractory angina pectoris, which appears to decrease myocardial ischaemia without masking symptoms, as shown by both clinical and experimental research. However, a large scale, multicentre randomised controlled trial is now necessary so that the effects of neurostimulation can be rigorously tested.

1 Murray S, Carson KGS, Ewings PD, et al. Spinal cord stimulation (SCS) significantly decreases the need for acute hospitalisation due to chest pain in patients with refractory angina pectoris. Heart 1999;82:89-92

2 Schofield PM, Sharples LD, Caine N, et al. Transmyocardial laser revascularisation in patients with refractory angina: a randomised controlled trial. Lancet 1999;353:519-24.

3 Shealey CN, Mortimer JT, Reswick JB. Electrical inhibition of pain by stimulation of the dorsal columns. Anesth Analg 1967;46:45-7.

4 Jacobs MJ, Jorning PJ, Joshi SR, et al. Epidural spinal cord electrical stimulation improves microvascular blood flow in severe limb ischaemia. Ann Surg 1988;207:170-83.

5 Mannheimer C, Carlsson C-A, Emanuelsson H, et al. Transcutaneous electrical nerve stimulation in severe angina pectoris. Eur Heart f 1982;3:297-302.

6 Mannheimer C, Carlsson CA, Emanuelsson H, et al. The Mannheimer C, Carlsson CA, Emanuelsson $\mathrm{H}$, et al. The peffects of transcutaneous electrical nerve stimulation in patients

7 Murphy DF, Giles KE. Dorsal column stimulation for pain relief from intractable angina pectoris. Pain 1987;28:365-8.

8 Mannheimer C, Augustinsson LE, Carlsson CA, et al. Epidural spinal electrical stimulation in severe angina pectoris. Br Heart f 1988:59:56-61.

9 Sanderson JE, Brooksby P, Waterhouse D, et al. Epidural spinal electrical stimulation for severe angina: a study of its effect on symptoms, exercise tolerance and degree of ischaemia. Eur Heart f 1992;13:628-33.

10 Dejongste MJL, Hautvast RWM, Hillege JL, et al on behalf of the Working Group on Neurocardiology. Efficacy of spinal cord stimulation as adjuvant therapy for intractable angina pectoris. F Am Coll Cardiol 1994;23:1592-7.

11 Sanderson JE, Ibrahim B, Waterhouse D, et al. Spinal electrical stimulation for intractable angina-long term clinical trical stimulation for intractable angina-long term

12 Dejongste MJL, Haaksma J, Hautvast RWM, et al. Effects of spinal cord stimulation on myocardial ischaemia during daily life in patients with severe coronary artery disease. A prospective ambulatory electrocardiographic study. $\mathrm{Br}$ Heart f 1994;71:413-18

13 Kujacic V, Eliasson T, Mannheimer C, et al. Assessment of spinal cord stimulation (SCS) on left ventricular function in patients with severe angina pectoris: an echocardiographic study. Eur Heart $\mathcal{F}$ 1993;14:1238-44.

14 Mannheimer C, Eliasson T, Andersson B, et al. Effects of spinal cord stimulation in angina pectoris induced by pacing and possible mechanisms of action. BMF 1993;307: 477-80.

15 Andersen C, Hole P, Oxhoj H. Does pain relief with spinal ord stimulation for angina conceal myocardial infarction? Br Heart F 1994;71:413-8.

16 Wall P. The placebo effect: an unpopular topic. Pain 1992;51:1-3.

17 Lasagna L, Mosteller F, Felsinger J, et al. A study of the placebo response. Am f Med 1954;16:770-9.

18 Hautvast RWM, DeJongste MJL, Staal MJ, et al. Spinal cord stimulation in intractable angina pectoris: A randomized, controlled efficacy study. Am Heart f 1998;136:1114-20.

19 Khurmi N, Bowles M, Kohli R, et al. Does placebo improve indexes of effort-induced myocardial ischaemia? An objective study in 150 patients with chronic stable angina pectoris. Am f Cardiol 1986;57:907-11

20 Chandler MJ, Brennan TJ, Garrison DW, et al. A mechanism of cardiac pain suppression by spinal cord stimulation: implications for patients with angina pectoris. Eur Heart f 1993;14:96-105.

21 Collins P, Fox KM. Pathophysiology of angina. Lancet 1990;335:94-6.

22 Eliasson T, Mannheimer C, Waagstein F, et al. Myocardial turnover of endogenous opioids and calcitonin-gene related peptide in the human heart and the effects of spinal cord stimulation on pacing induced angina pectoris. Cardiology 1998;89:170-7.

23 Mannheimer C, Emanuelsson H, Larsson G, et al. Influence of naloxone on the effects of transcutaneous electrical nerve stimulation (TENS) in pacing induced angina pectoris. Br Heart f 1994;71:419-21.

24 Falcone C, Specchia G, Rondanelli R, et al. Correlation between beta-endorphin plasma levels and anginal symptoms in patients with coronary artery disease. $7 \mathrm{Am}$ Coll Cardiol 1988;11:719-23.

25 Segal R, Murali S, Tipton K. Treatment of chronic unstable angina pectoris: use of a totally implantable programmable device for continuous intrathecal infusion of opiates: case report. Neurosurgery 1996;38:385-8.

26 Chauhan A, Mullins PA, Thuraisingham SI, et al. Effect of transcutaneous electrical nerve stimulation on coronary blood flow. Circulation 1994;89:694-702

27 Sanderson JE, Woo KS, Chung HK, et al. The effect of transcutaneous electrical nerve stimulation on coronary and systemic haemodynamics in syndrome X. Coron Artery Dis 1996;7:547-52.

28 Jessurun GAJ, Tio RA, DeJongste MJL, et al. Coronary blood flow dynamics during transcutaneous electrical nerves stimulation for stable angina pectoris associated with severe narrowing of one major coronary artery. $A m \mathcal{F}$ Cardiol 1998;82:921-6.

29 Ganz P, Braunwald E. Coronary blood flow and myocardial ischaemia. In: Braunwald E, ed. Heart disease, 5th ed. Philadelphia. WB Saunders, 1997:1161-3.

30 Hautvast RWM, Blanksma PK, DeJongste MJL, et al. Effect of spinal cord stimulation on regional myocardial blood flow assessed by positron emission tomography. Am $\mathcal{F} \mathrm{Car}-$ diol 1996;77:462-7.

31 De Landsheere C, Mannheimer C, Habets A, et al. Effect of spinal cord stimulation on regional myocardial perfusion assessed by positron emission tomography. Am $\mathcal{F}$ Cardiol 1992;69:1143-9.

32 Kaada B. Vasodilatation induced by transcutaneous nerve stimulation in peripheral ischaemia. Eur Heart $\mathcal{F} 1982 ; 3$ : 303-6.

33 Emanuelsson H, Mannheimer C, Waagstein F, et al. Catecholamine metabolism during pacing induced angina pectoris and the effect of transcutaneous electrical nerve stimulation. Am Heart F 1987;114:1360-6.

34 Wettervik C, Claes G, Drott C, et al. Endoscopic ransthoracic sympathicocotomy for severe angina. Lancet 1995;345:97-8.

35 Sanderson JE, Tomlinson B, Lau MJW, et al. The effects of transcutaneous nerve stimulation (TENS) on the autonomic nervous system. Clin Auton Res 1995;5:81-4.

36 Norsell H, Eliasson T, Mannheimer C, et al. Effects of pacing induced myocardial stress and spinal cord stimulation ing induced myocardial stress and spinal cord stimulation Heart F 1997; 18:1890-6.

37 Crea F, Pupita G, Galassi A, et al. Effect of theophylline on myocardial ischaemia. Lancet 1989;i:683-6.

38 Gaspardone A, Crea F, Iamele $M$, et al. Bamiphylline improves exercise-induced myocardial ischaemia through a novel mechanism of action. Circulation 1993;88:502-8.

39 Ferguson RJ, Petitclerc R, Choquette G, et al. Effect of physical training on treadmill exercise capacity, collateral circulation and progression of coronary disease. Am f Cardiol 1974;34:764-9.

40 Seiler C, Kaufmann U, Meier B. Intracoronary demonstration of adenosine induced coronary collateral steal. Heart 1997;77:78-81. 